\title{
Specific features of luminescence of oxygen-deficient centres in nanostructured silicon dioxide
}

\author{
V.S. Kortov, A.F. Zatsepin*, V.A. Pustovarov, A.A. Chudinov, D. Yu. Biryukov \\ Department of Physics and Technology, Ural State Technical University, Ekaterinburg 620002, 19 Mira St., Russia
}

Received 20 December 2006; accepted 1 February 2007

\begin{abstract}
Specific features of radiative and non-radiative relaxations of oxygen-deficient centres (ODC's) in nanostructured silicon dioxide were studied using optically stimulated electron emission and time-resolved photoluminescence. It was found that modifications of oxygen-deficient centres in the form of surface analogues can exist in nanostructured samples prepared by thermal decomposition of polysilazane in air. Photoluminescence of these centres was efficiently excited in the optical absorption bands of surface $E_{\mathrm{s}}^{\prime}$-centres and silicon clusters $\equiv$ SiSiSi $\equiv$ and could be associated with the intercentre energy transfer during their nonradiative relaxation. Specific features of thermally induced changes in the luminescence characteristics of the defects due to transformation of the structure of silica samples from amorphous to partially crystalline modification were established from analysis of the spectral composition and the decay kinetics of the photoluminescence.
\end{abstract}

(C) 2007 Published by Elsevier Ltd.

Keywords: Nanostructured silicon dioxide; Oxygen-deficient centres; Silicon clusters

\section{Introduction}

Functional properties of materials for luminescent detectors, sensors and transformers of ionizing radiation are formed mainly by structural defects. However, some problems related to the origin of defects and properties of excited states in silicon dioxide are still unclear. Moreover, electronic and optical characteristics of point defects in low-dimensional modifications of $\mathrm{SiO}_{2}$ are practically not investigated.

Models and luminescence characteristics of point defects in crystalline, amorphous, and thin-film modifications of silica still remain a subject of intensive investigation in connection with the high technological potential of these materials. The electronic structure and optical properties of such defects as $E^{\prime}$-centres, peroxide radicals, and nonbridging oxygen centres may be considered as reliably established (Skuja, 1998).

Research interest has recently been focused on luminescent oxygen-deficient centres. The silicon oxygen-deficient centres exhibit two luminescence bands at 2.7 and $4.4 \mathrm{eV}$ and two

\footnotetext{
* Corresponding author. Tel.: +7343 375 9788; fax: +73433754415.

E-mail address: zats@dpt.ustu.ru (A.F. Zatsepin).
}

excitation bands at 5.05 and about $7.50 \mathrm{eV}$ (Awazu et al., 1991). Germanium-doped silica glasses contain germanium oxygendeficient centres characterized by two luminescence bands at 3.2 and $4.3 \mathrm{eV}$ and two excitation bands at 5.15 and about $7.50 \mathrm{eV}$. Ultraviolet luminescence with fast decay $(\tau<5 \mathrm{~ns})$ was assigned to singlet-singlet transitions in oxygen-deficient centres. The luminescence observed at energies of $2.7 \mathrm{eV}(\tau \approx$ $10 \mathrm{~ms}$ ) and $3.2 \mathrm{eV}$ ( $\tau \approx 125 \mu \mathrm{s})$ with slow decay is attributed to the triplet-singlet transitions in silicon and germanium oxygendeficient centres, respectively.

The objective of this study was to obtain information about specific features of radiative and non-radiative relaxations of the oxygen-deficient centres (ODC's) in nanostructured silicon dioxide.

\section{Sample preparation and experimental techniques}

Our experiments were performed using $\mathrm{SiO}_{2}$ samples prepared by the thermal decomposition (pyrolysis) of polysilazane $\left(\mathrm{H}_{x} \mathrm{C}_{y} \mathrm{~N}_{z} \mathrm{Si}\right)$. Heating of polysilazane to $T=600^{\circ} \mathrm{C}$ for $48 \mathrm{~h}$ in air resulted in the chemical reaction

$\mathrm{H}_{x} \mathrm{C}_{y} \mathrm{~N}_{z} \mathrm{Si} \rightarrow \mathrm{SiO}_{2}+$ gaseous phase. 
The $\mathrm{SiO}_{2}$ samples prepared by this method actually were nanostructured porous ceramics. The examination of the surface of these samples in a scanning electron microscope revealed the presence of characteristic microstructured regions with a cross size of 100-500 nm, which probably appeared during the formation of the gas phase.

Additional samples of the $\mathrm{SiO}_{2}$ ceramic were annealed in air at temperatures of $1000^{\circ} \mathrm{C}$ (for $6 \mathrm{~h}$ ) and $1400^{\circ} \mathrm{C}$ (for $6 \mathrm{~h})$. According to helium picnometry, the skeletal densities of the samples were $2.42 \mathrm{~g} / \mathrm{cm}^{3}$ before annealing and 1.89 and $1.40 \mathrm{~g} / \mathrm{cm}^{3}$ after annealing, respectively. These data suggested that the heat treatment caused substantial transformations in the microstructure of the samples, which, in turn, led to the increase in their porosity. The X-ray powder diffraction analysis showed that the $\mathrm{SiO}_{2}$ samples were amorphous before annealing and contained some crystalline phase after annealing.

The structural state of the samples was thoroughly examined by the Raman spectroscopy at $100-2000 \mathrm{~cm}^{-1}$ at a Renishaw Raman microscope-spectrometer.

The optically stimulated electron emission (OSEE) spectra were measured over a wavelength interval of $200-600 \mathrm{~nm}$ at $295 \mathrm{~K}$. The UV radiation was produced by a DDS-30 deuterium lamp, whose spectrum was used to isolate fluxes of photons having the desired energy in a DMP-4 monochromator. The OSEE was measured in vacuum at a pressure of $10^{-4} \mathrm{~Pa}$ using a VEU-6 secondary-electron multiplier. The OSEE spectral responses obtained in our experiments were normalized to the source light flux.

The photoluminescence and photoluminescence excitation spectra at $T=8.5 \mathrm{~K}$ were measured under pulsed synchrotron radiation in the SUPERLUMI experimental station on the beamline of the HASYLAB laboratory at DESY (Hamburg, Germany). Excitations at $4.5-20.0 \mathrm{eV}$ were provided by a 2 -m vacuum monochromator. The photoluminescence excitation spectra were normalized to an equal number of photons incident on a sample. The photoluminescence spectra in the range $1.5-5.0 \mathrm{eV}$ were analysed with an ARC Spectra Pro-308i monochromator and an R6368P photomultiplier tube. The photoluminescence and photoluminescence excitation spectra were measured for the luminescence emitted in the time gate $\Delta t_{1}=14 \mathrm{~ns}, \Delta t_{2}=68 \mathrm{~ns}$ correlated with the excitation pulses of synchrotron radiation. The gate delay referred to the beginning of the synchrotron radiation pulse was $\delta t_{1}=1.9 \mathrm{~ns}, \delta t_{2}=17 \mathrm{~ns}$. The choice of the gating scheme was based on the preliminary analysis of the luminescence decay parameters.

\section{Result and discussion}

In order to elucidate the energy structure of the luminescent defects and to better understand the mechanisms responsible for their radiative relaxation, we measured the photoluminescence and photoluminescence excitation spectra at $T=8.5 \mathrm{~K}$. Fig. 1 displays the photoluminescence spectra measured for the $\mathrm{SiO}_{2}$ sample at $1.7-5.5 \mathrm{eV}$ under synchrotron radiation excitation in the range of $6.7-6.8 \mathrm{eV}$. The photoluminescence spectrum (Fig. 1) exhibits an intense band with a maximum at $3.1 \mathrm{eV}$ and a weaker band with a maximum at 2.7, 3.6 and $4.28 \mathrm{eV}$.

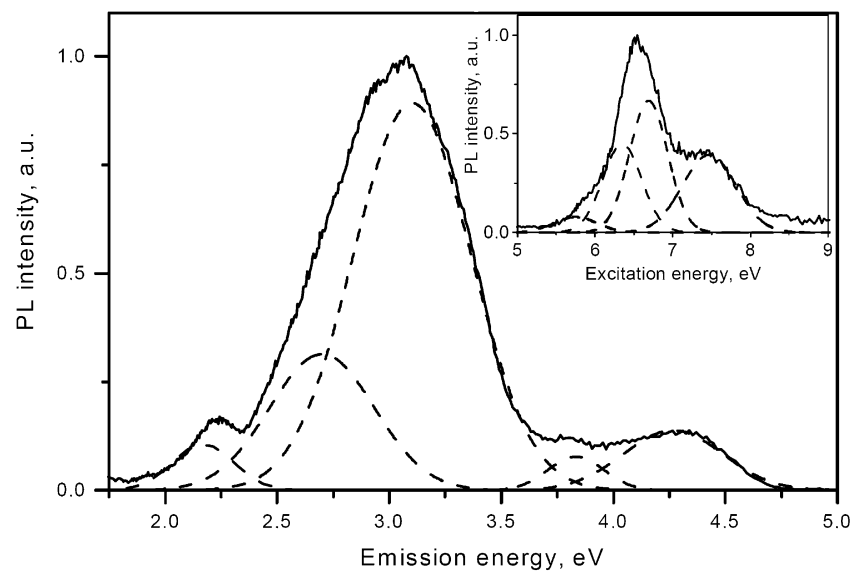

Fig. 1. Photoluminescence spectra of nanostructured silica samples, measured under excitation by $6.7 \mathrm{eV}$ photons. The inset shows the excitation spectra of luminescence at energies of $4.28 \mathrm{eV}$. The measurement temperature is $8.5 \mathrm{~K}$.

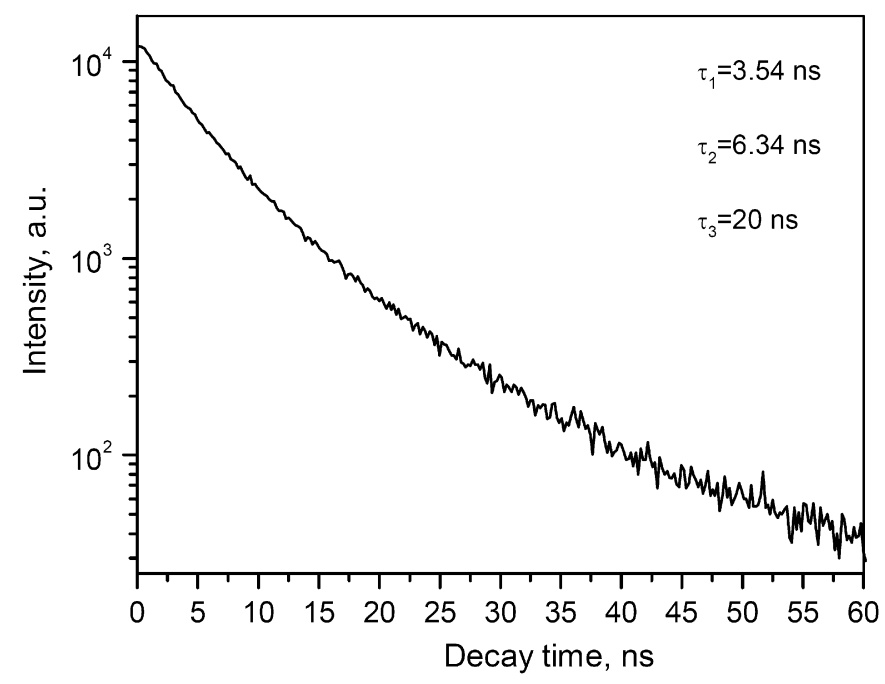

Fig. 2. Decay kinetics of the photoluminescence measured at $T=295 \mathrm{~K}$, $h v_{\mathrm{ex}}=6.36 \mathrm{eV}, h v_{\mathrm{em}}=4 \mathrm{eV}$ for nanostructured silica samples.

The excitation spectrum of photoluminescence at $4.28 \mathrm{eV}$ contains the two intense bands at 6.3 and $6.7 \mathrm{eV}$, as well as the weaker bands at 5.7 and $7.5 \mathrm{eV}$ (Fig. 1). The photoluminescence bands at 2.7 and $3.1 \mathrm{eV}$ and the photoluminescence excitation band at about $5.0 \mathrm{eV}$ are well known for amorphous modifications of silica and can be assigned to silicon and germanium oxygen-deficient centres, respectively (Agnello et al., 2003).

The observation of these bands in the photoluminescence and photoluminescence excitation spectra of this sample suggests the formation of oxygen-deficient centres (Skuja, 1998). At the same time, PL spectra contained fast-luminescence bands at 3.6 and $4.28 \mathrm{eV}$ (the decay constant $\tau \sim 7 \mathrm{~ns}$ ), which were related to the singlet-singlet luminescence $\left(S_{0} \rightarrow S_{1}\right)$ of different kinds of ODC's. Fig. 2 presents an example of the decay kinetics of the photoluminescence in the silica samples. Radiative transitions from excited triplet states $\left(\mathrm{T}_{1} \rightarrow \mathrm{S}_{0}\right)$ of ODC's showed up as luminescence bands at 2.7 and $3.1 \mathrm{eV}$. The shift of the maxima of the singlet and triplet PL bands in the 


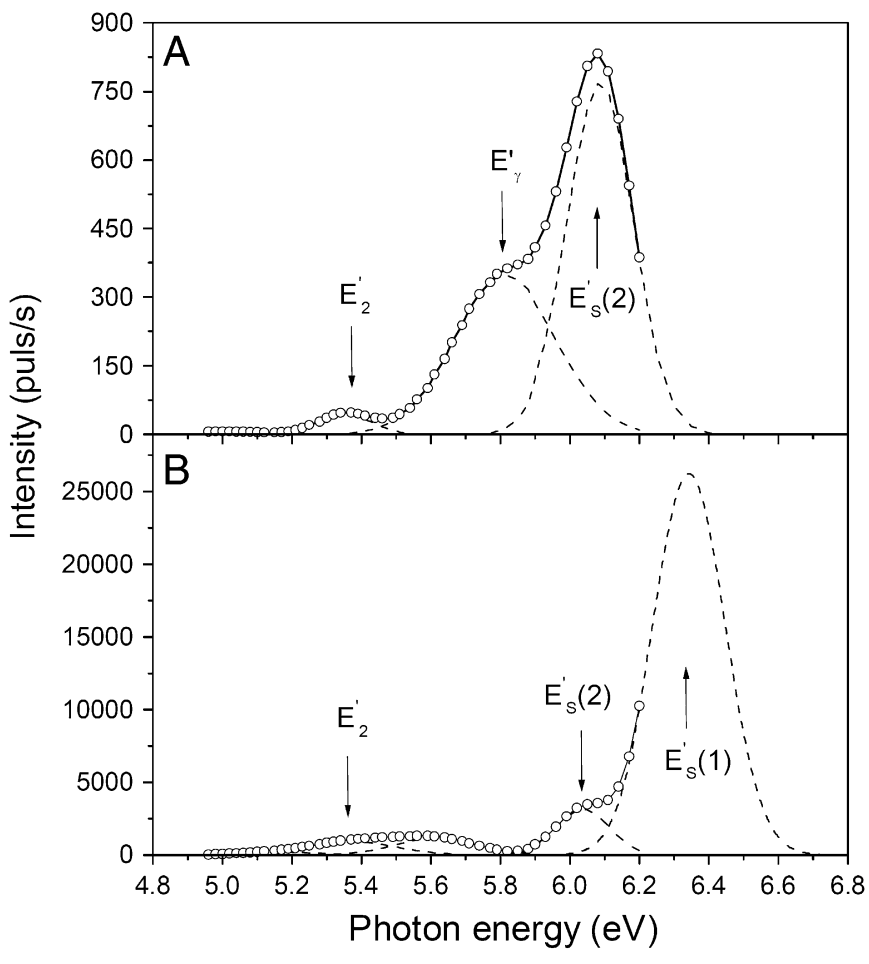

Fig. 3. OSEE spectra of radiation centres of nanostructured silica measured at $T=295 \mathrm{~K}$. (a) Sample annealed at $600^{\circ} \mathrm{C}$, (b) Sample after annealing at $1400^{\circ} \mathrm{C}$.

spectra of nano- $\mathrm{SiO}_{2}$ relative to those of the analogous bands in glassy $\mathrm{SiO}_{2}$ was explained by specific features of the ODC's local structure in low-dimensionality samples.

Specific features of the electronic structure of luminescent centres in the sample can originate, in particular, from the developed inner surface of the $\mathrm{SiO}_{2}$ nanoceramic. Generally, the experimental data suggest that the luminescence bands at 2.7 and $3.1 \mathrm{eV}$ in the spectrum of the sample can be attributed to surface oxygen-deficient centres. This suggestion is buttressed by the fast photoluminescence decay kinetics of these centres. A similar situation was observed earlier in the luminescence of surface defects in nanostructured germania and alumina (Zatsepin et al., 2005).

One more specific feature of the photoluminescence of these centres in nanostructured silica is that the excitation spectrum exhibits intense bands at 6.3 and $6.7 \mathrm{eV}$. The first band coincides with the well-known optical absorption band of surface $E^{\prime}$-centres, whose luminescence has not been detected so far (Skuja, 1998). Therefore, the excitation mechanism of luminescence of oxygen-deficient centres in this case can involve the intercentre excitation energy transfer between surface defects of different origins.

Information about non-radiative processes in $E^{\prime}$-centres can be obtained by the OSEE method. OSEE spectra of the samples of nano-silicon dioxide shown in Fig. 3 contained bands of bulk $E^{\prime}$-centres of two species $E_{2}^{\prime}$ and $E_{\gamma}^{\prime}$. The spectrum of the sample, which was treated at a temperature of $600^{\circ} \mathrm{C}$, was dominated by surface centres $E_{\mathrm{s}}^{\prime}(2)$ with a hydroxyl group in the nearest environment of the defect. The OSEE spectra of the sample, which was treated at a temperature of $1400^{\circ} \mathrm{C}$, were dominated by surface centres $E_{\mathrm{s}}^{\prime}(1)$ representing isolated three-coordinate silicon atoms with an unpaired electron. It was assumed that these centres could be located on the surface of internal microscopic pores in the test sample.

The spectral position of the band at $6.7 \mathrm{eV}$ corresponds to the optical excitation of oxygen-deficient centres to the highenergy singlet state $S_{2}$ (Agnello et al., 2003). At the same time, the excitation band at $6.7 \mathrm{eV}$ in the spectrum of nano-silica also correlates with the optical absorption of $\equiv \mathrm{SiSiSi} \equiv$ silicon clusters $(6.7 \mathrm{eV})$ revealed in nonstoichiometric silica glasses (Awazu et al., 1991). These observations suggest that additional excitation channels of the luminescence of oxygen-deficient centres can exist in the materials under study.

Heat treatment of the silica sample at temperatures of 1000 and $1400^{\circ} \mathrm{C}$ caused a considerable transformation of the energy structure of the luminescent centres. It manifested itself as the shift of the photoluminescence bands and led to the obvious acceleration of slow (triplet-singlet $\mathrm{T}_{1} \rightarrow \mathrm{S}_{0}$ ) and retardation of fast (singlet-singlet $S_{0} \rightarrow S_{1}$ ) radiative relaxation processes.

\section{Conclusion}

The results of the analysis of the optically stimulated electron emission and the photoluminescence suggest the formation of different modifications of oxygen-deficient centres. The photoluminescence of these centres in the absorption bands of surface centres and $\equiv \mathrm{SiSiSi} \equiv$ clusters can be excited by the energy transfer during non-radiative relaxation of $E_{\mathrm{s}}^{\prime}$-centres and silicon nanoclusters. The study of the luminescence spectra and the radiative relaxation decay kinetics revealed specific features of the conversion of luminescent defects.

Moreover, an obvious tendency to acceleration of slow (triplet-singlet) and retardation of fast (singlet-singlet) radiative processes was observed after thermal treatment of nano- $\mathrm{SiO}_{2}$. Thus, the obtained results point to the possibility of a purposeful change of luminescent properties of $\mathrm{SiO}_{2}-$ nanostructures that can be useful for modification of technical characteristics of radiation detectors.

\section{Acknowledgements}

This study was supported by RFBR (Projects Nos. 04-0296073, 05-02-16448 and 05-02-16530) and the RF Ministry of Education (Project RNP.2.1.1.2948).

\section{References}

Agnello, S., Boscaino, R., Cannas, M., Gelardi, F.M., Leone, M., Boizot, B., 2003. Competitive relaxation processes of oxygen deficient centres in silica. Phys. Rev. B 67, 0333202-2.

Awazu, K., Kawazoe, H., Muta, K., 1991. Optical properties of oxygen deficient centers in silica glasses in $\mathrm{H}_{2}$ or vacuum ambient. J. Appl. Phys. $70,60-62$.

Skuja, L., 1998. Optically active oxygen-deficiency-related centers in amorphous silicon dioxide. J. Non-Cryst. Solids 239, 16-48.

Zatsepin, A.F., Kortov, V.S., Pustovarov, V.A., Biryukov, D.Yu., 2005. Timeresolved spectroscopy of radiation defects in nanocrystalline germanium dioxide. Phys. Status Solidi 2 (1), 343-346. 